\title{
SISTEMA DE BAIXO CUSTO PARA EXECUÇÃO E MONITORAMENTO ON-LINE DE REAÇÕES FOTOCATALÍTICAS: APLICAÇÃO EM REDUÇÃO DE NITRO-FENOL
}

\author{
Byanca S. Salvatia , Sirlon F. Blaskievicz ${ }^{\mathrm{a}}$, Patricia G. Corradini ${ }^{\mathrm{a}, \mathrm{b}}$ e Lucia H. Mascaro ${ }^{\mathrm{a}, *,([)}$ \\ a'Departamento de Química, Universidade Federal de São Carlos, 13565-905 São Carlos - SP, Brasil \\ 'Instituto Federal de Educação, Ciência e Tecnologia Fluminense, 28300-000 Itaperuna - RJ, Brasil
}

Recebido em 26/03/2021; aceito em 10/06/2021; publicado na web em 06/07/2021

\begin{abstract}
LOW-COST SYSTEM FOR ONLINE MONITORING OF PHOTOCALYTICAL REACTIONS: APPLICATION IN NITROPHENOL. There is a global concern with pollutants and organic contaminants and efficient and inexpensive methods are sought for the treatment of these toxic products. Generally, large volume reactors and the use of expensive equipment are required to monitor a photoreaction. The present work proposes the construction of a colorimetric equipment, which allows the online monitoring of photoreactions, using low-cost materials that allow the analysis from small aliquots. To show the applicability of the system, we studied the reduction reaction of 4-nitrophenol (4NP) with $\mathrm{NaBH}_{4}$. The impedimetric sensor enabled to construct the kinetic curve of the decrease in the chromophore band. It was possible to observe that the 4NP reduction reaction depends on: (i) chromophore concentration; (ii) stability of the reducing agent and; (iii) repulsion between the anions. The system developed here opens doors, as it can be used using a photocatalyst in the form of film, and it has also proved to be an inexpensive alternative for both research and teaching laboratories, as it allows the execution of chemical physics practices such as kinetic monitoring of reactions and mathematical adjustment of the curves.
\end{abstract}

Keywords: impedimetric sensor; online monitoring; photoreduction; remediation of organic contaminants.

\section{INTRODUÇÃo}

A presença de poluentes prioritários e contaminantes orgânicos emergentes, incluindo produtos farmacêuticos, pesticidas, hormônios, surfactantes e vários compostos em águas superficiais e no solo, é uma preocupação global. ${ }^{1}$ Metodologias eficazes e baratas para o monitoramento e tratamento para remoção de produtos orgânicos tóxicos contaminantes, ou em uma conversão em produtos de valor agregado, são cada vez mais estudadas e valorizadas. ${ }^{2}$

Dentre os resíduos industriais e agrícolas que se buscam converter em compostos de menor toxicidade, destaca-se os compostos nitro aromáticos. O 4-nitro fenol (4NP), é um exemplo desta classe que, mesmo em concentrações baixas $\left(0,1 \mathrm{mg} \mathrm{kg}^{-1}\right)$, pode atuar como disruptor endócrino, promover irritação dérmica e ocular, e apresentar potencial carcinogênico. ${ }^{3,4}$ Muitos estudos se dedicam a avaliar processos oxidativos baseados em métodos fotoquímicos, ${ }^{5,6}$ visando a conversão a espécies não-agressivas ou até à completa mineralização desses compostos. ${ }^{2}$ Mas uma alternativa ao manejo do composto 4NP tem ganho destaque: a fotorredução, que possa gerar compostos de interesse industrial, como 4-aminofenol (4AP), utilizado como precursor do paracetamol.

Devido à alta estabilidade química, a redução de 4NP normalmente é promovida por metais nobres, tornando o processo menos viável do ponto de vista econômico. Semicondutores também podem ser combinados a catalisadores metálicos, e o uso de agentes de sacrifício como hidrazina $\left(\mathrm{NH}_{2} \mathrm{NH}_{2}\right)$, ou os íons boro hidreto $\left(\mathrm{BH}_{4}^{-}\right)$ e sulfito $\left(\mathrm{SO}_{3}^{-2}\right)$ auxiliam a reação. A redução catalítica de $4 \mathrm{NP}$ pelo ânion $\mathrm{BH}_{4}^{-}$tornou-se um dos modelos para avaliar a atividade de um fotocatalisador. Embora a reação seja termodinamicamente possível, é cineticamente restrita pela repulsão dos ânions boro hidreto e nitro fenolato, ${ }^{7}$ e o que supera essa barreira é a afinidade de adsorção dos ânions na superfície do catalisador, fornecendo os elétrons necessários para complementar a reação. ${ }^{8}$

\footnotetext{
*e-mail: 1mascaro@ufscar.br
}

Urkude et $a l .{ }^{9}$ conseguiram reduzir efetivamente o nitro à amino sem utilizar nanopartículas metálicas, somente empregando um compósito de $\mathrm{TiO}_{2}$ e polianilina com $\mathrm{NaBH}_{4}$ como agente de sacrifício, utilizando radiação visível. Semicondutores que absorvem a radiação visível como é o caso do $\mathrm{BiVO}_{4}$ são preferíveis, visando o uso da energia solar como fonte de radiação.

Outro problema muito comum que afeta a viabilidade de acompanhamento dessas reações, especialmente em aspectos cinéticos, é a infraestrutura instrumental. ${ }^{10,11}$ A montagem de sistemas de valor mais acessível pode trazer contribuições relevantes para o estudo de materiais, sem falar na possibilidade de aplicação na formação de recursos humanos, podendo ser utilizado em aulas práticas, como uma ferramenta auxiliadora no processo de ensino e aprendizagem. Além da elaboração deles, com materiais de baixo custo, muitas vezes considerado lixo eletrônico, ${ }^{12}$ o entendimento dos princípios mencionados permite a miniaturização do equipamento e, por consequência, do sistema reacional empregado.

Neste trabalho, o uso de um sensor impedimétrico LDR (Light Dependent Resistor), que é de fácil acesso e baixo custo, foi utilizado para acompanhamento da fotorreação de 4-nitrofenol. Uma cubeta de quartzo foi utilizada como um fotorreator, sem necessidade de retirar alíquotas. A reação foi acompanhada por um multímetro, e o sistema foi validado, com um auxílio de outras técnicas.

\section{PARTE EXPERIMENTAL}

\section{Montagem do sistema}

\section{Ideia do sistema de detecção}

O sensor LDR é um componente cuja resistência varia linearmente com a intensidade da luz: quanto maior a quantidade de luz incidente, menor a resistência observada. Ao utilizar um diodo emissor de luz (LED) com radiação na faixa de absorção do analito, ou seja, um cromóforo que absorva parte desta radiação, haverá uma mudança na resposta do detector. Assim, será possível obter uma relação 
linear entre a concentração deste cromóforo e a resistência lida no multímetro através de uma constante de proporcionalidade (eq. 1). Dessa forma, é possível a construção de curvas de concentração ou até mesmo curvas cinéticas de uma reação onde se tem a variação do cromóforo de interesse em função do tempo.

$$
\frac{I}{I_{0}}=\alpha c
$$

em que $\mathrm{I}_{0}$ é a intensidade do sinal (resistência) na ausência do analito, que se refere a máxima transmitância possível, I é a intensidade na presença do analito, $\alpha$ é a constante de proporcionalidade e c a concentração.

\section{Montagem experimental}

A Figura 1 ilustra a montagem do sistema. Uma espuma rígida foi utilizada para fazer um suporte para cubeta, cortando-a nas dimensões mostradas na Figura 1S (disponível no Material Suplementar-MS), com auxílio de um estilete. Esse suporte foi fixado com fita adesiva sob um agitador magnético. Na parte superior do suporte o sensor LDR foi inserido e atado com conector bipolar para que fosse possível encaixar o multímetro posteriormente. Utilizando-se um suporte universal e garra, fixou-se o sistema de iluminação (LED M405L2 Thorlabs - UK), com comprimento de onda $405 \mathrm{~nm}$, verificado pelo espectro de emissão, apresentado na Figura $2 \mathrm{~S}$ (disponível no MS). Com um radiômetro aferiu-se a potência da luz para $100 \mathrm{~mW} / \mathrm{cm}^{2}$ e, nesta distância $(\sim 10 \mathrm{~cm})$ fixou-se o agitador com o suporte. Um multímetro foi conectado ao sensor LDR para que fosse possível fazer a leitura da resistência. A Figura 3S (disponível no MS) apresenta uma fotografia do aparamento experimental montado.

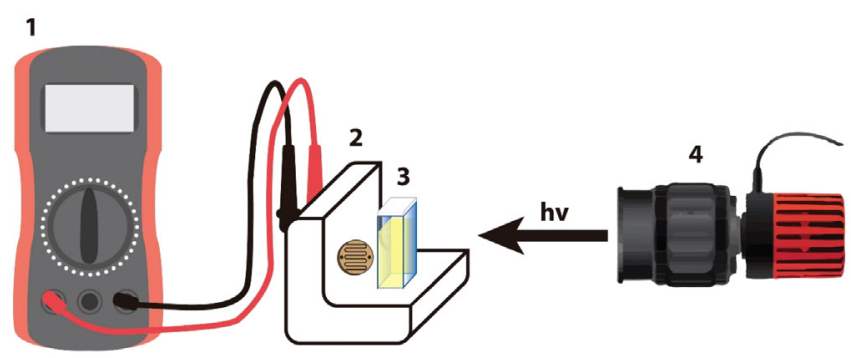

Figura 1. Esquema representativo do funcionamento do protótipo de colorímetro: (1) multímetro para coleta de dados; (2) suporte com o LDR e a (3) cubeta com analito e (4) fonte de radiação

\section{Acompanhamento da reação de fotorredução do nitro-fenol}

Para a escolha da fonte de iluminação mais adequada para o monitoramento da concentração do 4NP, um espectro de UV-vis do $4 \mathrm{NP}$ protonado e na forma de ânion fenolato foi coletado. A Figura 2 mostra um espectro na faixa de 200 a $600 \mathrm{~nm}$, para soluções de 4NP em pH 5,5 (majoritariamente na forma fenol) e 8,5 (majoritariamente na forma fenolato). Visto que a forma desprotonada do 4NP tem uma banda predominante na região de $400 \mathrm{~nm}$, optou-se por utilizar o LED $405 \mathrm{~nm}$ (M405L2 Thorlabs - UK) como fonte de radiação.

Com o sistema pronto, fez-se uma curva de calibração do p-nitrofenol $\left(24-50 \mu \mathrm{mol} \mathrm{L}{ }^{-1}\right)$. Utilizou-se cinco pontos de concentrações, a partir de uma solução estoque de nitrofenol $\left(5,0 \times 10^{-4} \mathrm{~mol} \mathrm{~L}^{-1}\right)$, diluindo em tampão fosfato $0,01 \mathrm{~mol} \mathrm{~L}^{-1}$, ajustado para $\mathrm{pH} 7,5$, com soluções estoque de $\mathrm{HCl}$ e $\mathrm{KOH}$. Em todos os pontos, o valor da resistência da solução foi acompanhado. Espectros de absorção também foram obtidos em um espectrômetro UV-vis comercial (Varian, Cary 5G UV-vis-NIR) para comparação com o sensor do protótipo.

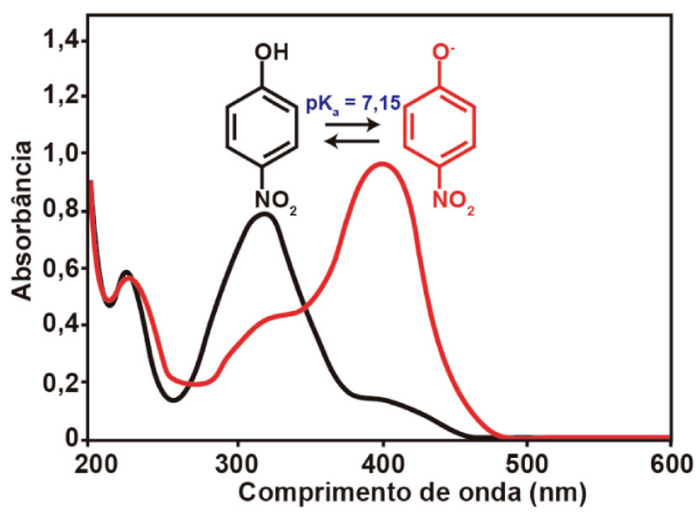

Figura 2. Espectro de UV-vis do $4 N P$ nas formas de fenol e fenolato

\section{Fotólise dos reagentes}

Após a calibração do sensor, a fotólise do nitrofenol foi realizada. Em uma cubeta, adicionou-se $0,5 \mathrm{~mL}$ da solução estoque de 4NP e 2,5 mL de tampão bicarbonato, ajustado em $\mathrm{pH} 8,0$. A cubeta foi colocada no sistema com o agitador ligado para garantir a homogeneidade da solução, e então, o LED foi ligado e as medidas de resistência foram anotadas a cada 30 minutos até 360 minutos. Foram feitos espectros pré e pós reação no equipamento comercial.

A fotólise do reagente $\mathrm{NaBH}_{4}$ também foi avaliada. Em uma cubeta, adicionou-se $3,0 \mathrm{~mL}$ de tampão bicarbonato ajustado em pH 9,0 e 1,0.10-4 mol de $\mathrm{NaBH}_{4}$. A cubeta foi colocada no agitador com LED $405 \mathrm{~nm}$, acompanhando a resistência durante 60 minutos. Espectros de absorção UV-vis da solução foram realizados antes e após reação.

\section{Estudo de $\mathrm{pH}$}

A fotorredução do 4NP foi monitorada em $\mathrm{pH} 6,7,8$ e 9. Usouse tampão fosfato para realização da medida em pH 6 e 7 e tampão bicarbonato para os demais valores de $\mathrm{pH}$; os ajustes foram feitos com soluções estoque de $\mathrm{HCl}$ e $\mathrm{KOH}$. Em todos os experimentos colocou-se em uma cubeta $0,5 \mathrm{~mL}$ da solução estoque de $4 \mathrm{NP}$ e 2,5 mL de tampão com $\mathrm{pH}$ ajustado. A cubeta foi colocada no sistema sob agitação e uma pequena alíquota de solução de $\mathrm{NaBH}_{4}$ $\left(1,0 \times 10^{-5} \mathrm{~mol} \mathrm{~L}^{-1}\right)$ foi adicionada ao sistema. Os valores da resistência da solução foram acompanhados pelo tempo de reação e medidas de espectroscopia de absorção na região UV-vis foram realizados antes e após a reação.

No pH 8,0, a reação também foi feita no escuro, acompanhada em UV-vis utilizando-se o programa "Scanning Kinetics". Também foi realizada uma reação da mesma forma descrita anteriormente, mas usando no lugar do LED um simulador solar acoplado a um filtro (O-56), que corta a radiação abaixo de $560 \mathrm{~nm}$.

\section{Estudo com LED de menor potência}

Com o intuito de verificar se a mesma reação poderia ser mediada por um LED de menor potência, que tornaria possível diminuir ainda mais o custo do sistema, fez-se a reação de p-nitrofenol com $\mathrm{NaBH}_{4}$ em pH 8,0, substituindo-se o LED $405 \mathrm{~nm}$ por um LED comercial comum (LED alto brilho azul, $408 \mathrm{~nm}$ ). O espectro de emissão deste LED está apresentado na Figura 2S do MS. O LED foi fixado em um quadrado de espuma rígida e ligado a uma fonte de alimentação $6 \mathrm{~V}$ (carregador de celular), inserindo um resistor de $100 \Omega$ entre a fonte e o LED. O suporte com LED foi acoplado ao suporte com sensor LDR utilizando-se tarraxas. Antes dessa reação, testou-se uma curva de calibração nas mesmas condições adotadas para o LED $405 \mathrm{~nm}$. 


\section{RESULTADOS E DISCUSSÃO}

\section{Calibração do sistema LDR}

A curva de calibração do p-nitrofenol obtido pelo protótipo do colorímetro apresentou linearidade, como apresentado na Figura 3(a). $O$ coeficiente de determinação linear foi de $r^{2}=0,98$, indicando uma boa correlação entre o sinal obtido e a faixa de concentração analisada. A título de comparação, a mesma curva de concentração foi realizada em espectrômetro de UV-Vis, pela intensidade de absorbância em $400 \mathrm{~nm}$ (Figura 3(b)). O coeficiente obtido pelo espectrômetro foi semelhante ao obtido pelo protótipo do colorímetro, destacando a boa aplicação do sistema desenvolvido.
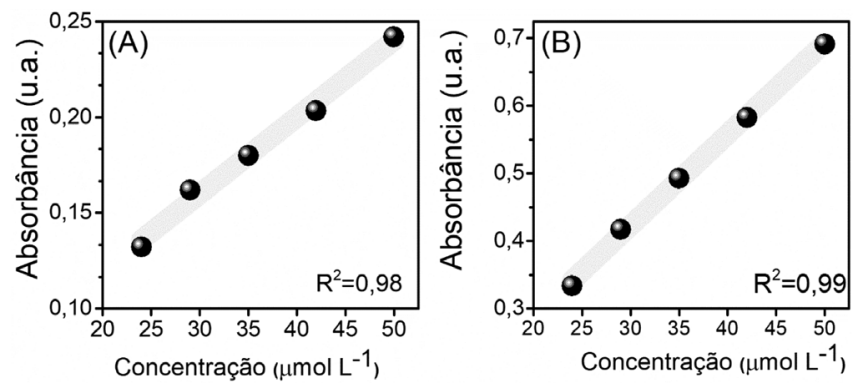

Figura 3. (A) Curva de calibração 4NP utilizando sensor colorimérico do protótipo; (B) curva de calibração do $4 N P$ utilizando espectrômetro UV-vis

\section{Fotólise das substâncias}

Um possível caminho da redução do 4NP é gerar o aminofenol (4AP). Para avaliar se os compostos $4 \mathrm{NP} \mathrm{NaBH}_{4}$ não apresentariam fotólise, acompanhou-se o sistema por $60 \mathrm{~min}$ em iluminação. Os valores obtidos no monitoramento da fotólise do 4NP estão apresentados na Figura 4S, no MS. No caso do $\mathrm{NaBH}_{4}$, como a solução não mostrou nenhuma banda na região de emissão do LED, não foi possível o acompanhamento pelo sensor.

Para confirmação da fotoestabilidade dos reagentes, medidas de absorção de UV-Vis foram realizadas nas amostras, antes e após a iluminação, conforme representado na Figura 4. Na espectroscopia de UV, a banda de absorção em $300 \mathrm{~nm}$ pode indicar o 4AP, enquanto a banda em $400 \mathrm{~nm}$ indica absorção do nitrofenolato. ${ }^{8} \mathrm{Na}$ fotólise do p-nitrofenol, obteve-se uma degradação quase imperceptível da banda em $400 \mathrm{~nm}$, indicando que qualquer reação que ocorra estaria sendo bem lenta (Figura 4(a)). Descartou-se também influência da luz na degradação do $\mathrm{NaBH}_{4}$, uma vez que a sua fotólise não apresentou qualquer banda notável no UV (Figura 4(b)).
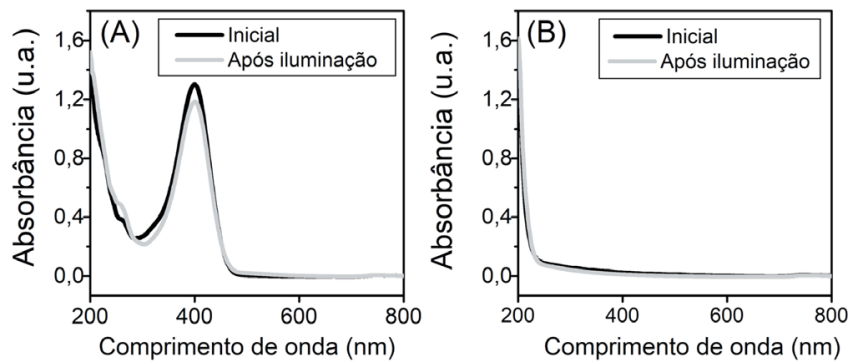

Figura 4. Gráfico de absorbância por comprimento de onda antes e após $1 \mathrm{~h}$ de iluminação em $400 \mathrm{~nm}$ para (A) e $50 \mu \mathrm{mol} \mathrm{L} \mathrm{L}^{-1}$ de 4-nitrofenol em solução tampão de bicarbonato ajustado $\mathrm{pH}=8$, (B) $100 \mathrm{\mu mol} \mathrm{L}^{-1}$ de $\mathrm{NaBH}_{4}$ em solução tampão de bicarbonato ajustado em $\mathrm{pH}$ 9,0

\section{Estudo cinético da fotorredução de 4-nitrofenol}

Após realizar a correlação de concentração de 4NP com a resistência da solução do sistema e descartar a hipótese de autofotólise dos compostos estudados, iniciaram-se os estudos cinéticos. Os ajustes iniciais mostraram um comportamento de primeira ordem da reação, assim, os ajustes matemáticos foram realizados levando em consideração a lei das velocidades integradas da seguinte forma:

$$
\frac{d[4 N P]}{d t}=-k_{o b s}[4 N P]
$$

Reajustando a expressão (2) tem-se:

$$
\frac{d[4 N P]}{[4 N P]}=-k_{o b s} d t
$$

Integrando a expressão (3) do tempo inicial ao tempo " $t$ " se obtém a expressão:

$$
[4 N P]=[4 N P]_{0} e^{-k_{o b s} t}
$$

em que [4NP] é a concentração de 4NP no tempo "t", [4NP $]_{0}$ é a concentração inicial de $4 \mathrm{NP}, \mathrm{k}_{\mathrm{obs}}$ é a constante velocidade de pseudoprimeira ordem e t é o tempo. Por fim, substituindo a concentração da expressão (1) na expressão (4), obtém-se a seguinte expressão em função da absorbância:

$$
I=I_{0} e^{-k_{o b s} t}
$$

\section{Efeito de $\mathrm{pH}$}

Em meio aquoso, o 4-nitrofenol e seu ânion apresentam duas formas de ressonância (Figura 2), benzenóide e quinonóide, onde uma carga negativa está localizada no grupo nitro. ${ }^{13} \mathrm{O}$ aumento do $\mathrm{pH}$ do meio modifica a distribuição dessas espécies, promovendo a desprotonação do grupo hidroxila, e a ressonância da forma quinonóide (desprotonada) é a responsável pela absorção da radiação na região de $400 \mathrm{~nm}$ (cor amarela). ${ }^{13}$ Assim, para avaliar a influência da desprotonação da substância 4NP, acompanhou-se a reação com o sistema homemade nos pHs 6,0, 7,0, 8,0 e 9,0. Para avaliar a possível formação de produtos, medidas de absorção por UV-vis foram realizadas nas soluções nas condições iniciais e após a reação. O sinal-resposta para cada condição e os respectivos espectros de absorção de UV-Vis estão apresentados na Figura 5.

De maneira a extrair os parâmetros cinéticos da reação, as curvas obtidas em diferentes $\mathrm{pH}$ foram ajustadas pela equação (4), uma vez que dados da literatura indicam que a reação de fotorredução do 4NP segue comportamento de pseudo-primeira ordem. ${ }^{14} \mathrm{~A}$ expressão aqui deduzida pode ser inserida em softwares como o Origin $9^{\circledR}$ e realizar o ajuste matemático diretamente pela equação exponencial, uma vez que a linearização, apesar das facilidades atreladas, pode diminuir a precisão de um ajuste. ${ }^{15}$

Nos estudos cinéticos da influência do $\mathrm{pH}$ notou-se que no $\mathrm{pH}$ 6,0 (Figura 5 (a) e (b)), houve uma pequena diminuição da banda do $4 \mathrm{NP}$, porém sem formar banda em $300 \mathrm{~nm}$. Possivelmente isso ocorreu porque o $\mathrm{NaBH}_{4}$ se degrada muito rapidamente em $\mathrm{pH}$ mais baixos. O tempo de meia vida da hidrólise espontânea do $\mathrm{NaBH}_{4}$ pode ser calculado pela seguinte equação. ${ }^{16}$

$$
\log _{10} t_{1 / 2}=p H-(0,034 T-1,92)
$$

em que $\mathrm{t}_{1 / 2}$ é o tempo de meia vida, em minutos e T é a temperatura, em Kelvin.

O tempo de meia vida do composto em pH 6 está na ordem de frações de segundos, aumentando exponencialmente em função do pH. ${ }^{16}$ No pH 7,0 (Figura 5 (c) e (d)), os resultados foram parecidos 
com estes também, com uma melhora na degradação em $400 \mathrm{~nm}$ e um discreto aparecimento de banda em $300 \mathrm{~nm}$.

Em pH 8,0 (Figura 5 (e) e (f)), a completa degradação do 4NP e a formação da possível banda de aminofenol foram obtidas. Nesse $\mathrm{pH}$, mais de $50 \%$ do $4 \mathrm{NP}$ presente encontra-se na forma quinonóide, visto que o $\mathrm{pK}_{\mathrm{a}}$ da espécie é 7,15. Além de uma maior quantidade do cromóforo, o tempo de meia vida do $\mathrm{NaBH}_{4}$ já aumenta para aproximadamente $40 \mathrm{~s}$, favorecendo a reação pela maior absorção em $400 \mathrm{~nm}$ e pelo maior tempo de meia vida de $\mathrm{NaBH}_{4}$ no meio.

No pH 9,0 (Figura 5 (g) e (h)), é possível notar que, assim como no $\mathrm{pH} 8,0$, há uma contribuição do $\mathrm{pH}$ mais alto na excitação do cromóforo, porém aqui, provavelmente ocorre também uma repulsão de ânions do cromóforo com os do boro-hidreto. Nota-se, com isso, que a reação é termodinamicamente possível, porém cineticamente dificultada pela ocorrência da repulsão das cargas. ${ }^{17,18}$
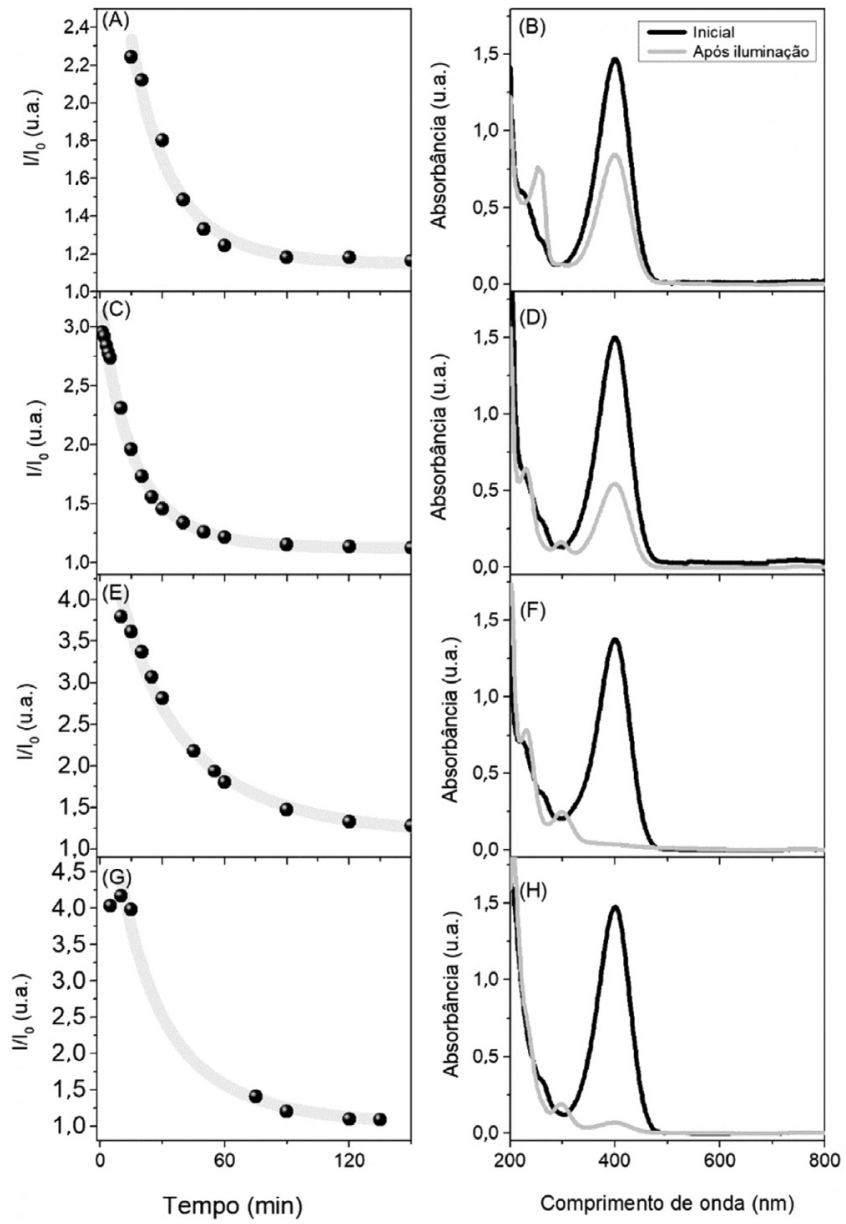

Figura 5. Gráfico da relação sinal resposta em função do tempo da reação e respectivos espectros $U V$-Vis inicial e pós reação do $4 N P$ em $A, B$ ) pH 6,0; C,D) $\mathrm{pH} 7,0 ; \mathrm{E,F}$ ) $\mathrm{pH} 8,0$ e $G, H) \mathrm{pH} 9,0$

A Tabela 1 apresenta os valores das constantes velocidade de pseudo-primeira observadas para a reação de fotorredução de 4-nitrofenol em diferentes valores de $\mathrm{pH}$. Como observado nos espectros completos apresentados na Figura 5, não necessariamente o consumo do $4 \mathrm{NP}$ ocorrerá por completo em todos os valores de pH. Em algumas situações a hidrólise espontânea do $\mathrm{NaBH}_{4}$ pode competir com a reação de redução do $4 \mathrm{NP}$. Em suma, existem 3 fatores que explicam as constantes de velocidade obtidas: (i) o tempo de meia vida do $\mathrm{NaBH}_{4}$; (ii) a concentração do 4NP na forma desprotonada (cromóforo) e (iii) a repulsão entre os íons fenolato e borohidreto. Em valores de $\mathrm{pH}$ mais baixos, o tempo de meia vida do íon borohidreto é exponencialmente reduzido e a reação atinge um patamar devido a decomposição desta espécie, e não pelo final da reação com o 4NP, o que foi confirmado fazendo novas adições de $\mathrm{NaBH}_{4}$ no pH sob iluminação e observando o decaimento do sinal até a completa extinção da banda em $400 \mathrm{~nm}$, mostrada na Figura $5 \mathrm{~S}$ do MS. Com isso, a constante de velocidade obtida acaba sendo, no caso do $\mathrm{pH}$ 6, uma medida indireta do consumo do $\mathrm{NaBH}_{4}$. O mecanismo completo da reação ainda precisa ser investigado em trabalhos futuros, porém, propõe-se que ela se inicie devido a uma foto ativação do cromóforo, ${ }^{19}$ assim, com o aumento do $\mathrm{pH}$ também há um aumento da concentração do cromóforo do 4NP, que faria com que a reação ocorresse por completo e de maneira mais rápida, porém, como visto no $\mathrm{pH}$ 9, a repulsão entre os ânions acaba impedindo que a reação acontecesse por completo, com isso o pH 8 acabou se mostrando a melhor relação entre os 3 fatores citados.

Tabela 1. Valores das constantes velocidade de pseudo-primeira ordem $\left(\mathrm{k}_{\mathrm{obs}}\right)$ observadas para a fotorredução de 4NP em diferentes pHs

\begin{tabular}{ll}
\hline $\mathrm{pH}$ & $\mathrm{k}_{\mathrm{obs}}\left(\mathrm{s}^{-1}\right)$ \\
\hline 6,0 & $6,6 \cdot 10^{-4}$ \\
7,0 & $7,4 \cdot 10^{-4}$ \\
8,0 & $3,3.10^{-4}$ \\
9,0 & $4,0.10^{-4}$ \\
\hline
\end{tabular}

Para verificar se a reação foi um efeito de excitação do cromóforo pelo LED emitindo em $405 \mathrm{~nm}$, realizou-se a mesma reação no escuro (Figura 6(a)), e não foi observado nenhum consumo da banda em $400 \mathrm{~nm}$, apenas uma pequena flutuação na absorbância. Ao analisar em função do tempo, esta flutuação mostrou-se ser um indicativo da influência do ânion borohidreto, deslocando levemente o $\mathrm{pH}$ do meio, porém, atenuado pelo tampão utilizado. Ao iluminar a cubeta com radiação acima de $560 \mathrm{~nm}$ (Figura 6(b)), também por 2 horas não foi possível observar a mesma variação de bandas que ao iluminar com o LED405, indicando que a reação ocorre majoritariamente por uma foto excitação do cromóforo do 4NP. Ainda, como a iluminação estava fora da região de absorção do 4NP, nesse caso não foi possível monitorar a concentração do cromóforo pelo sensor LDR.
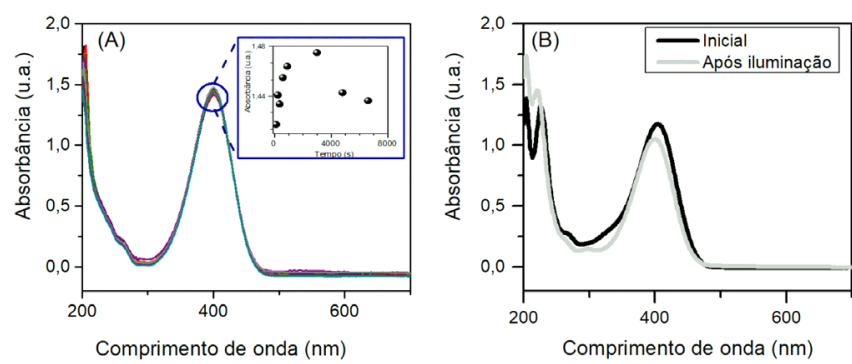

Figura 6. Espectros de UV-Vis da reação do $4 \mathrm{NP}$ em pH $8 \mathrm{~A}$ ) ao longo do tempo e sem iluminação, a variação da banda em $400 \mathrm{~nm}$ em função do tempo está apresentada no inset do gráfico; B) Inicial e após iluminação acima de $560 \mathrm{~nm}$

\section{Estudo com LED de menor potência}

No intuito de baratear ainda mais o sistema, optou-se por realizar a reação do $4 \mathrm{NP}$ com $\mathrm{NaBH}_{4}$ utilizando um LED comum, que custa menos de R \$1,00. De acordo com fabricante, a emissão é em $408 \mathrm{~nm}$, que é próximo ao relatado por outros trabalhos na literatura. ${ }^{20}$ Para validar esse sistema, foi feita uma nova curva de calibração com o 4NP (apresentada na Figura 6S do MS), que assim como no LED 405 apresentou uma boa linearidade na relação sinal/resposta $\left(\mathrm{R}^{2}=0,98\right)$, mostrando-se adequada para o monitoramento do 4NP. Ao realizar 
a reação, entretanto, o 4NP se comportou da mesma maneira que quando a reação foi realizada no escuro (Figura 6(a)). Ao medir a irradiância do LED, essa se encontrava em torno de $100 \mu \mathrm{W} \mathrm{cm}{ }^{-2}$, 1000x menor do que a densidade de potência utilizada com o LED 405. Isso indica que a potência do LED comum não é suficiente para ativar reações fotoquímicas, apesar de ser eficiente para o monitoramento colorimétrico como demonstrado aqui e em outros trabalhos da literatura. ${ }^{21,22}$

\section{CONCLUSÕES}

Neste trabalho foi possível o desenvolvimento de um sistema que, de maneira eficiente aproveita a fonte de iluminação de uma reação fotocatalítica para o monitoramento de um cromóforo (4NP). Um ponto a ressaltar é que esse sistema permite trabalhar com volumes reacionais reduzidos em relação a reatores convencionais, gerando uma economia dos reagentes envolvidos, bem como a detecção online ainda torna possível manter esse volume de trabalho constante. Ainda são necessários estudos, valendo-se de outras técnicas para uma elucidação concreta do mecanismo da reação do $4 \mathrm{NP}$ com $\mathrm{NaBH}_{4}$, porém, combinando os dados obtidos no sensor do protótipo com espectros obtidos em equipamento de UV-Vis comercial, foi possível inferir que, dependendo do $\mathrm{pH}$, a reação possivelmente conduzia ao produto de fotorredução 4AP. O sensor impedimétrico empregado permitiu construir a curva cinética da diminuição da banda do cromóforo 4NP em $405 \mathrm{~nm}$, e observou-se que esta reação depende de 3 principais fatores: (i) Concentração do cromóforo; (ii) Estabilidade do agente redutor e; (iii) Repulsão entre os ânions. Com o aumento do $\mathrm{pH}$, o equilíbrio fenol/fenolato é deslocado para a forma aniônica do 4NP, responsável pela absorção na faixa de luz utilizada, e muito provavelmente a reação se dê pela excitação dessa molécula, visto que não ocorreu ao ser iluminada com radiação de maior comprimento de onda. Ainda, apesar da decomposição do íon boro hidreto em meio aquoso também diminuir com o aumento do $\mathrm{pH}$, a repulsão aniônica entre os reagentes faz com que um valor intermediário $(\mathrm{pH} 8)$ seja um ponto otimizado para a reação completa. Por fim, notou-se que o LED utilizado também aquecia o sistema, porém, em um estudo do efeito da temperatura atingida por esse sistema, a reação no escuro teve mudanças pouco significativas. $\mathrm{O}$ sistema aqui desenvolvido abre portas, pois pode ser empregado utilizando um fotocatalisador na forma de filme, e também se mostrou uma alternativa barata tanto para laboratórios de pesquisa, quanto de ensino, pois permite a execução de práticas de físico-química como o acompanhamento cinético de reações e o ajuste matemático das curvas.

\section{MATERIAL SUPLEMENTAR}

Algumas imagens dos sistemas utilizados neste trabalho estão disponíveis em http://quimicanova.sbq.org.br, na forma de arquivo PDF, com acesso livre.

\section{AGRADECIMENTOS}

Os autores agradecem à Coordenação de Aperfeiçoamento de Pessoal de Nível Superior (CAPES) - Código Financeiro 001 e ao Conselho Nacional de Pesquisa e Desenvolvimento, pelas bolsas concedidas. Os autores também agradecem à Fundação de Pesquisa de São Paulo (FAPESP) pela assistência financeira ao projeto e pelas bolsas concedidas (2018/16401-8, 2014/50249-8, 2013/07296-2).

\section{REFERÊNCIAS}

1. Uyguner-Demirel, C. S.; Birben, N. C.; Bekbolet, M.; Catal. Today 2017, 284, 202.

2. Kefeni, K. K.; Mamba, B. B.; Sustainable Mater. Technol. 2020, 23, 140.

3. Bae, S.; Gim, S.; Kim, H.; Hanna, K.; Appl. Catal., B 2016, 182, 541.

4. Li, C.; Taneda, S.; Suzuki, A. K.; Furuta, C.; Watanabe, G.; Taya, K.; Toxicol. Appl. Pharmacol. 2006, 217, 1.

5. Zhou, M.; Wu, Z.; Zhu, J.; Ye, Q.; Fu, J.; Chin. J. Catal. 2002, 23, 376.

6. Malinowska, B.; Walendziewski, J.; Robert, D.; Weber, J.; Stolarski, M.; Appl. Catal., B 2003, 46, 441.

7. Huang, J.; Vongehr, S.; Tang, S.; Lu, H.; Meng, X.; J. Phys. Chem. C 2010, 114, 15005.

8. Hernández-Gordillo, A.; Romero, A. G.; Tzompantzi, F.; Oros-Ruiz, S.; Gómez, R.; J. Photochem. Photobiol., A 2013, 257, 44.

9. Urkude, K.; Thakare, S. R.; Gawande, S.; J. Environ. Chem. Eng. 2014, 2,759 .

10. Burgess, A. E.; Davidson, J. C.; J. Chem. Educ. 2014, 91, 300.

11. Benito-Lopez, F.; Verboom, W.; Kakuta, M.; Gardeniers, J. H. G.; Egberink, R. J.; Oosterbroek, E. R.; van den Berg, A.; Reinhoudt, D. N.; Chem. Commun. 2005, 2857.

12. Mercer, C.; Leech, D. N.; J. Chem. Educ. 2017, 94, 816.

13. Muniz-Miranda, M.; Appl. Catal., B 2014, 146, 147.

14. Singh, J.; Mehta, A.; Rawat, M.; Basu, S.; J. Environ. Chem. Eng. 2018, $6,1468$.

15. Zeviani, W. M.; Dissertação de mestrado, Universidade Federal de Lavras, Lavras, Brasil, 2009.

16. Richardson, B. S.; Birdwell, J. F.; Pin, F. G.; Jansen, J. F.; Lind, R. F.; J. Power Sources 2005, 145, 21.

17. Fahrenbach, A. C.; Bruns, C. J.; Li, H.; Trabolsi, A.; Coskun, A.; Stoddart, J. F.; Acc. Chem. Res. 2014, 47, 482.

18. Tomsho, J. W.; Benkovic, S. J.; J. Org. Chem. 2012, 77, 2098.

19. Den Heijer, J.; Shadid, O.; Cornelisse, J.; Havinga, E.; Tetrahedron 1977, 33, 779.

20. Rabello, B. R.; Caetano, W.; Quim. Nova 2018, 41, 920.

21. Reis, R. A.; Dissertação de mestrado, Universidade Estadual Paulista, Brasil, 2014.

22. Silva, V. B.; Orth, E. S.; Quim. Nova 2017, 40, 238. 